\title{
Cuantificación Económica de los Residuos de Construcción de una Edificación en Altura: Un Caso de Estudio
}

\section{Jorge Bravo ${ }^{1}$, Claudia Valderrama ${ }^{1}$ y Felipe Ossio ${ }^{1 *}$}

(1) Pontificia Universidad Católica de Chile, Facultad de Ingeniería, Escuela de Construcción Civil, Vicuña Mackenna 4860, Macul, Santiago, Chile. (e-mail: jebravo2@uc.cl; c.valderrama@uc.cl; faossio@uc.cl)

${ }^{*}$ Autor a quien debe ser dirigida la correspondencia

Recibido Jun. 20, 2018; Aceptado Ago. 21, 2018; Versión final Oct. 19, 2018, Publicado Abr. 2019

\section{Resumen}

Este artículo presenta un estudio de evaluación económica y clasificación de residuos de construcción y demolición (RCD) en un proyecto real ubicado en la Región Metropolitana, Chile. Chile genera más de cinco millones y medio de toneladas de RC\&D al año y afectan de múltiples maneras el medio ambiente. A su vez, los RC\&D son un síntoma de problemas de productividad. Una adecuada gestión de los residuos requiere una cuantificación de los mismos. Sin embargo, se observa una carencia en la literatura relacionada a la cuantificación de los volúmenes de residuos y su impacto económico (generación, transporte y disposición final). Se encuentra que el volumen de residuos generados por superficie construida para este caso de estudio es de $0,186 \mathrm{~m}^{3} / \mathrm{m}^{2}$ de RCD con un costo total de $188.234,44$ US $\$$ correspondiente al $1,19 \%$ del presupuesto. Así mismo, el costo ponderado de la gestión del RCD se estimó en 75,47 US\$/m³.

Palabras clave: residuos de construcción y demolición; cuantificación económica; edificio

\section{Economic Assessment of the Construction Waste of a High- Rise Building: A Case Study}

\begin{abstract}
this article presents a study of economic assessment and classification of construction and demolition waste (CDW) in a high-rise building project located in the Metropolitan Region of Santiago, Chile Chile generates over five and half million tons of CDW per year and affect the environment in multiple ways. Likewise, the CDW are also a symptom of productivity problems. An adequate management of waste requires an assessment of it. However, there is limited literature related to the assessment of waste volumes and their economic impact (generation, transport and final disposal). It is found that the estimated waste generated per constructed area reached $0,186 \mathrm{~m}^{3} / \mathrm{m}^{2}$ for this study case with a total cost of $188.234,44$ US $\$$ that corresponds to $1.19 \%$ of the project budget. Likewise, the costs per volume of waste was $75,47 \mathrm{US} \$ / \mathrm{m}^{3}$.
\end{abstract}

Keywords: construction and demolition waste, economic assessment; buildings 


\section{INTRODUCCIÓN}

Los Residuos de Construcción y Demolición (RCD) afectan de múltiples maneras el medio ambiente: contaminan el suelo, el agua y el aire, cambian el entorno natural entre otras degradaciones del ecosistema. Se estima que en la Unión Europea (UE), los desechos de construcción y demolición llegan a los 180 millones de toneladas anuales (Gobierno de Chile, 2011). Los principales generadores de desechos son Alemania, Reino Unido, Francia, España y Países Bajos, éste último es el que tiene un mayor porcentaje de reutilización o reciclaje con un $90 \%$ (Trachte, 2003). Ejemplos más cercanos son México, donde solo considerando el Distrito Federal, hasta el año 2007 se generaban entre 3.000 y 4.000 toneladas de RCD diarios (Tam y Tam, 2006), o Colombia, quien, de acuerdo a la Secretaria Distrital de Planificación y Ambiente de Bogotá, existe una generación per cápita de 2 toneladas de residuos de construcción al año (Castaño et al., 2013). En Chile, el año 2009, el sector construcción produjo 5.821 .000 toneladas de RCD, cifra que corresponde al $34,4 \%$ de los residuos urbanos totales del país (Castillo y Ossio, 2012), lo que además trae consigo otra problemática que es la de disponer terrenos para su vertimiento o los altos costos que implica su manejo (Valdés y Rapimán, 2007 y Jin et al., 2017), de ahí la importancia de estimar económicamente su cuantificación en los proyectos.

A la fecha, los RCD han sido foco de diversos estudios e investigaciones, observándose un progresivo interés a partir de la década de los 90's, tal como muestra el artículo de Wu et al., (2014), quienes esquematizaron la literatura existente sobre materias de residuos proporcionando una fuente de información que permite comprender de mejor manera los modelos de cuantificación, clasificación y gestión de los mismos. Pese a lo anterior, la literatura recabada en ese documento no contempla tópicos donde se evalúen económicamente los RCD en un proyecto de edificación ni tampoco casos de éxito en clasificaciones de residuos enfocadas a la edificación en altura, debido a que es difícil determinar las cantidades y composición (Umar et al., 2018). Es así que la cuantificación de los residuos es considerada un requisito previo para la aplicación exitosa de la gestión de los RCD. En efecto, "se ha reconocido que la cuantificación adecuada de los residuos de construcción y demolición es de gran importancia para establecer un sistema de gestión eficaz tanto a nivel de proyecto como a nivel regional" (Wu et al., 2014). Y es por esa razón que, junto a la escasez de información económica de RCD, se detecta la necesidad de implementar un estudio que sea un aporte tanto para la comunidad de científicos e investigadores como para los profesionales del rubro quienes desarrollan análisis y tomas de decisión en sus proyectos.

El presente artículo, mediante el análisis de un caso de estudio, tiene por objetivo cuantificar la cantidad de residuos generados y, al mismo tiempo, evaluar económicamente el costo $/ \mathrm{m}^{2}$ que implica la generación, almacenamiento, transporte interno y retiro a disposición final de estos. El caso de estudio corresponde a un proyecto de edificación en altura de 21 pisos con una superficie útil de $13.375 \mathrm{~m}^{2}$ ubicado en un sector residencial de la Región Metropolitana (Chile). Wu et al., (2014) muestran en su revisión analítica de 57 investigaciones relativas a los RCD que, si bien la metodología de estimación de residuos ha sido fuente de interés, la evaluación económica de los mismos y su impacto en el presupuesto a raíz de la gestión, transporte y disposición final de estos son un tópico relativamente inexplorado. De ese modo, se origina un punto de partida para elaborar un catastro económico puesto que se identifica el método de construcción, tamaño del proyecto, tipo de edificio, método de almacenamiento de material, errores humanos y problemas técnicos como los principales factores de generación de residuos en construcciones nuevas (Wu et al., 2014). De igual manera, Aldana y Serpell (2012) realizan una selección de documentos mostrando los materiales más citados en la literatura de residuos de construcción (hormigón, madera, ladrillos, plástico, metal, acero y placas de yeso-cartón) y las metodologías de estimación de residuos de acuerdo con volumen, peso y valor financiero basado en cada metro cuadrado construido. Asimismo, Romnée y Vrijders (2017) establecen una base de datos con la tasa de desperdicios de los materiales más utilizados en proyectos monitoreados en Bruselas (Bélgica).

Solís-Guzmán et al., (2009) desarrollan un modelo de éxito implementado en la Comunidad Alcores (Sevilla) en España donde se calcula los volúmenes totales y subgrupos de los residuos: demolido, restos y desechos de empaque, mediante una clasificación sistémica de los materiales utilizados y cálculo de desechos esperados. Además, se observa en la literatura diversas metodologías de cuantificación, a saber: Inspección en Obra; Cálculo de tasa de generación; Análisis de Ciclo de Vida; Clasificación por Sistemas de Acumulación; Modelamiento Variable; entre otras, cuya elección dependerá de los recursos y nivel de detalle que se desee en la investigación (Wu et al., 2014 y Subramaniam et al., 2018). Por otro lado, en un estudio preliminar de residuos de la construcción en Antofagasta (Chile) se calculó que en edificación habitacional en altura por cada $\mathrm{m}^{2}$ construido se generan $0,220 \mathrm{~m}^{3}$ de residuos (Cámara Chilena de la Construcción, 2013). Para esto realizaron encuestas donde se obtuvo información esencial sobre los proyectos en ejecución de la región y se entrelazó con la base de datos generada por la Dirección de Obras Municipales (departamento del municipio encargado en velar el cumplimiento reglamentario de los proyectos de construcción). 
Es preciso señalar que a través de la literatura encontrada se muestra que existe variada información con respecto a los residuos de construcción, pero falta investigar el aspecto económico dentro de los proyectos. Y, de la misma manera, se evidencia la necesidad de indagar una evaluación económica especializada a una tipología en particular con el fin de conseguir antecedentes detallados que sirvan como insumo en las tomas de decisiones

\section{METODOLOGÍA}

El objetivo de la presente investigación es cuantificar la cantidad de residuos generados y, al mismo tiempo, evaluar económicamente el costo $/ \mathrm{m}^{2}$ que implica la generación, almacenamiento, transporte interno y retiro a disposición final de estos en un proyecto de construcción. Para ello, el estudio se basa en el análisis teórico de un proyecto residencial en altura, de 21 pisos, con una superficie útil de $13.375 \mathrm{~m}^{2}$ ubicado en la Región Metropolitana (Chile). Mediante el Método de Acumulación del Sistema de Clasificación (Wu et al., 2014) se realiza una cuantificación a nivel de diseño de los desechos de construcción esperados del proyecto. Método basado en el cálculo de residuos que relaciona una cantidad generada con una unidad específica (como $\mathrm{kg} / \mathrm{m}^{2}$ o $\mathrm{m}^{3} / \mathrm{m}^{2}$ ) que propone el Método de Cálculo de la Tasa de Generación (Wu et al., 2014). El método utilizado considera la información proyectada para calcular las pérdidas y residuos generados por los empaques de cada material, y la estimación de recursos materiales se relaciona con el sistema de clasificación propuesta en la Lista Europea de Residuos LER (Gobierno de España, 2002). Para ello se cuenta con la siguiente información: i) Presupuesto de obra, ii) Análisis de precios unitarios (APU) y iii) Cubicaciones.

De este modo, con la información antes señalada, se tabulan todos los recursos materiales utilizados en el proyecto. Para cada material involucrado se determina a qué partida y actividad pertenecen, su clasificación LER, cantidad de desechos generados, su unidad y factor de conversión a $\mathrm{m}^{3}$, y precio unitario. La cantidad generada de desechos corresponde a la estimación calculada desde el diseño del proyecto y los residuos generados por los envases o recipientes los cuales no necesariamente han reducido su volumen al momento de llevarlos a botadero. Se consideran las pérdidas de cada recurso material en base a valores de mercado dados por la media de proyectos anteriores y, para los envases se utilizan cantidades referidas a mediciones empíricas. Se determina la medición y cuantificación por volumen $\left(\mathrm{m}^{3}\right)$, ya que es la unidad comercial que se utiliza al momento de contratar servicios dentro de la gestión de residuos (como envío a botadero). Además, para el presente estudio las obras exteriores no son contabilizadas. Luego, se llevó a cabo la ponderación entre precio a) unitario, b) pérdidas y c) cantidades proyectadas, para posteriormente, desarrollar el análisis de las actividades que generan mayor volumen de residuos $\left(\mathrm{m}^{3}\right)$, cantidad total de residuos generados $\left(\mathrm{m}^{3}\right)$, relación de residuos generados por superficie del proyecto $\left(\mathrm{m}^{3} / \mathrm{m}^{2}\right)$ y, finalmente, el costo total de la gestión del residuo en dólares (US\$). Se considera el supuesto de un costo a botadero constante equivalente a 9,46 US $\$ / \mathrm{m}^{3}$ dado por la composición de $3,97 \mathrm{US} \$ / \mathrm{m}^{3}$ como costo fijo y $5,49 \mathrm{US} \$ / \mathrm{m}^{3}$ como costo variable correspondiente al traslado de $12 \mathrm{~km}$ de distancia.

\section{RESULTADOS Y DISCUSIÓN}

El estudio elaboró una base de datos con 1.627 elementos, cuyos residuos fueron clasificados según la Lista Europea de Residuos (Gobierno de España, 2002). La figura 1 da cuenta de los principales residuos esperando en el proyecto de estudio, que tal como se observa, no necesariamente corresponden a la mayor cantidad de materiales utilizados, dado que la generación de residuos varía de elemento a elemento. En efecto, el $85 \%$ de los residuos esperados se clasifican en tres categorías a saber: 170407 - Metales Mezclados (49\%); 170107 - Mezclas de hormigón, ladrillos, tejas y materiales cerámicos (20\%); y 170802 Materiales de construcción a base de yeso (16\%).

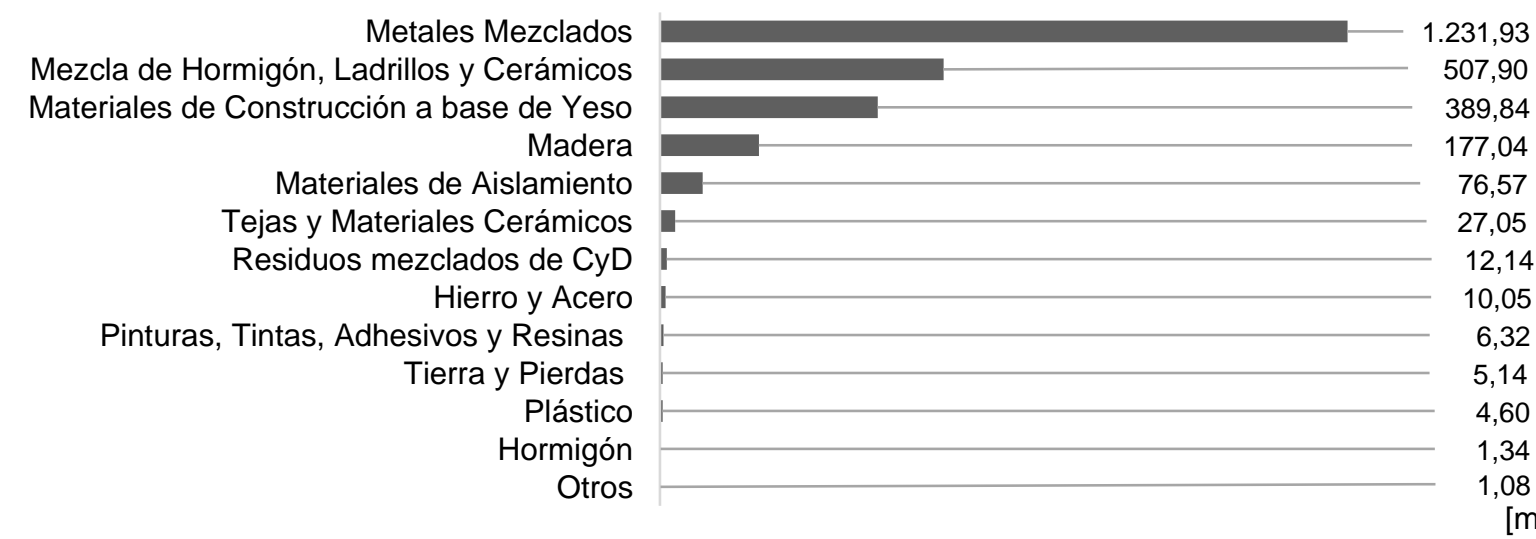

Fig. 1: Distribución residuos según clasificación LER 
Se determina que la generación teórica de residuos del proyecto de edificación en altura, de 21 pisos y uso residencial, es de $2.494 \mathrm{~m}^{3}$. Para este análisis se consideraron las etapas Obra Gruesa y Terminaciones. De este modo se dejan fueran las etapas Movimiento de Tierra, Instalaciones y Obras Exteriores, ya que son actividades que no generan residuos considerables y/o son subcontratadas a otras empresas quienes asumen la disposición final, por lo anterior no son parte del alcance del presente estudio.

\section{Obra Gruesa}

En Obra Gruesa se identificaron 140 recursos materiales distribuidos entre 8 partidas: Hormigón, Techumbre y hojalatería, moldajes, enfierradura, carpintería metálica, impermeabilización y sellos, y rellenos. Y, 46 actividades (relleno de ripio, enfierradura, hormigón, ente otros). La producción total de residuos teóricos generados en esta etapa corresponde a $545 \mathrm{~m}^{3}$. La Tabla 1 muestra el desglose de los residuos generados en las 8 grandes partidas identificadas. De estas la partida Hormigón concentra el $90 \%$ de los residuos generados en la etapa de Obra Gruesa. De las 46 actividades involucradas en estas grandes 8 partidas, se identifica que el hormigonado de losa concentra el $89 \%$ de los residuos proyectados, seguido muy de lejos por las actividades de estructura techumbre, moldaje y enfierradura sobre cota cero, con una participación de 3\% cada una (ver tabla 2).

Tabla 1: Generación de RCD por partida en obra gruesa.

\begin{tabular}{|l|c|c|}
\hline \multicolumn{1}{|c|}{ Partidas Obra Gruesa } & Residuos generados $\left[\mathrm{m}^{3}\right]$ & Residuos por unidad superficie $\left[\mathrm{m}^{3} / \mathrm{m}^{2}\right]$ \\
\hline Hormigón & 492,57 & 0,03683 \\
\hline Techumbre y Hojalatería & 18,22 & 0,00136 \\
\hline Moldajes & 15,33 & 0,00115 \\
\hline Enfierradura & 15,18 & 0,00113 \\
\hline Carpintería Metálica & 1,67 & 0,00012 \\
\hline Impermeabilización y Sellos & 1,18 & 0,00009 \\
\hline Rellenos & 0,65 & 0,00005 \\
\hline Total & 544,79 & 0,04073 \\
\hline
\end{tabular}

Tabla 2: Generación de RCD por actividad en obra gruesa.

\begin{tabular}{|l|c|c|}
\hline \multicolumn{1}{|c|}{ Actividades Obra Gruesa } & Residuos generados $\left[\mathrm{m}^{3}\right]$ & $\begin{array}{c}\text { Residuos por unidad } \\
\text { superficie }\left[\mathrm{m}^{3} / \mathrm{m}^{2}\right]\end{array}$ \\
\hline Hormigón Losa Edificio & 482,79 & 0,03610 \\
\hline Estructura Techumbre & 16,25 & 0,00121 \\
\hline Moldaje & 15,33 & 0,00115 \\
\hline Enfierradura Sobre Cota Cero Edificio & 15,18 & 0,00113 \\
\hline Base Grúa & 5,43 & 0,00041 \\
\hline Emplantillado & 4,35 & 0,00033 \\
\hline Aislación De Techumbres & 1,79 & 0,00013 \\
\hline Baranda Balcones Deptos. y Terrazas & 1,43 & 0,00011 \\
\hline Impermeabilización & 1,18 & 0,00009 \\
\hline Preparación Sub-Base en Radier y Losa Fundación & 0,47 & 0,00004 \\
\hline Otros & 0,60 & 0,00004 \\
\hline Total & 544,79 & 0,04073 \\
\hline
\end{tabular}

Al estimar el costo de los residuos, considerando tanto el precio de venta del elemento como el costo a botadero (valor referencial de 9,46 US $\$ / \mathrm{m}^{3}$ incluido transporte y disposición final) se identifica que tanto las partidas como las actividades más influyentes en generación de residuos concuerdan con las partidas (figura 2) y actividades (figura 3) más influyentes desde el punto de vista económico. Esto se explica dada la gran diferencia en la magnitud de residuos generados, siendo poco influyente el valor del elemento.

\section{Terminaciones}

En el caso de Terminaciones se identifican 1.487 recursos materiales distribuidos entre 43 grandes partidas y 272 actividades (tabiques tipo, aislación acústica, cerámica baños, entre otros). La producción total de residuos teóricos generados en esta etapa corresponde a $1.949 \mathrm{~m}^{3}$. 


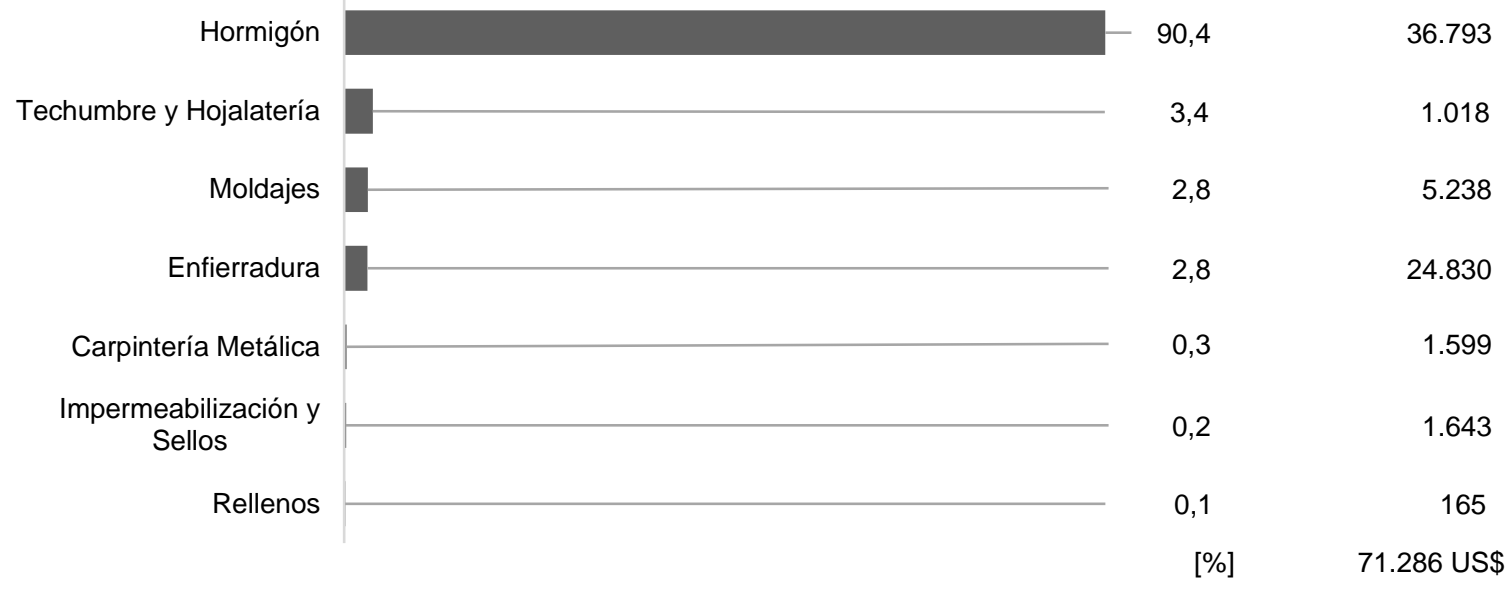

Fig. 2: Distribución de residuos generados por partida en obra gruesa

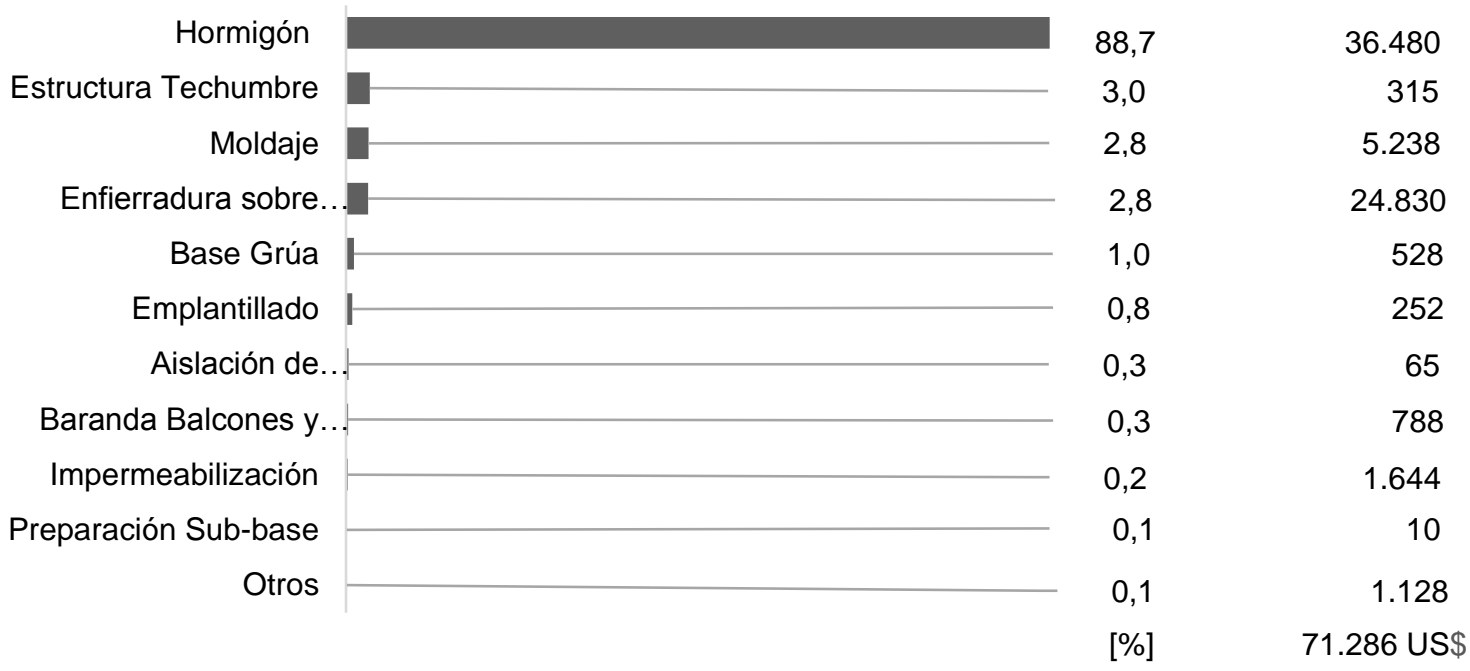

Fig. 3: Distribución de residuos generados por actividad en obra gruesa.

La Tabla 3 muestra el desglose de los residuos generados en las 10 partidas más influyentes. De estas, 3 partidas concentran el $92 \%$ de los residuos generados en la etapa de Terminaciones, a saber: Tabiquería Departamentos (58\%); Tabiquería Zonas Comunes (26\%); y Guardapolvos (8\%). De las 272 actividades involucradas en estas 43 partidas, se identifica que Tabiquería Volcometal concentra el $54 \%$ de los residuos proyectados, seguido muy de lejos por las actividades de Guardapolvo Recintos Departamento y Aseo de Terminaciones, con una participación de $7 \%$ y $2 \%$ respectivamente (ver tabla 4).

Tabla 3: Generación de RCD en las 10 partidas más relevantes de terminaciones.

\begin{tabular}{|l|c|c|}
\hline \multicolumn{1}{|c|}{ Partidas Relevantes Terminaciones } & Residuos $\left[\mathrm{m}^{3}\right]$ & Residuos $\left[\mathrm{m}^{3} / \mathrm{m}^{2}\right]$ \\
\hline Tabiquería en Dptos. & $1.129,31$ & 0,084 \\
\hline Tabiquería en Zonas Comunes & 499,68 & 0,037 \\
\hline Guardapolvos Dptos. & 165,38 & 0,012 \\
\hline Aseo Terminaciones & 36,25 & 0,003 \\
\hline Revestimiento Piso Dpto. & 21,30 & 0,002 \\
\hline Revestimiento Muros Dpto. & 19,18 & 0,001 \\
\hline Cornisas Zonas Comunes & 14,83 & 0,001 \\
\hline Enlucidos Mecanizados y Retapes Dptos. & 11,62 & 0,001 \\
\hline Sobrelosas y Aislación Losas & 10,25 & 0,001 \\
\hline Estucos & 9,58 & 0,001 \\
\hline Otros & 31,98 & 0,002 \\
\hline Total & $1.949,37$ & \\
\hline
\end{tabular}


Tabla 4: Generación de RCD en las 10 actividades más relevantes de terminaciones.

\begin{tabular}{|l|c|c|}
\hline \multicolumn{1}{|c|}{ Actividades Relevantes Terminaciones } & Residuos $\left[\mathrm{m}^{3}\right]$ & Residuos $\left[\mathrm{m}^{3} / \mathrm{m}^{2}\right]$ \\
\hline Tabiquería Volcometal & $1.058,43$ & 0,079 \\
\hline Guardapolvo Recintos Departamento & 142,37 & 0,011 \\
\hline Aseo De Terminaciones & 36,25 & 0,003 \\
\hline Dinteles Dpto. y F-15 Bodegas & 26,78 & 0,002 \\
\hline Junquillo Deptos. & 25,22 & 0,002 \\
\hline Cerámica & 25,17 & 0,002 \\
\hline Cornisas Hall Ascensores, Recintos y Circulaciones & 14,85 & 0,001 \\
\hline Enlucido Mecanizado Deptos. & 14,17 & 0,001 \\
\hline Parquet Hall Estar Comedor, Pasillos y Dormitorios & 10,46 & 0,001 \\
\hline Faldones de Tina Dpto. & 7,43 & 0,001 \\
\hline Otros & 588,23 & 0,044 \\
\hline Total & $1.949,37$ & 0,146 \\
\hline
\end{tabular}

Al estimar el costo de los residuos de la etapa Terminaciones, considerando tanto el precio de venta del elemento como el costo a botadero (valor referencial de 9,46 US $\$ / \mathrm{m}^{3}$ incluido transporte y disposición final) se constata, al igual que en Obra Gruesa, que tanto las partidas como las actividades más influyentes en generación de residuos concuerdan con las partidas (figura 4) y actividades (figura 5) más influyentes desde el punto de vista económico.

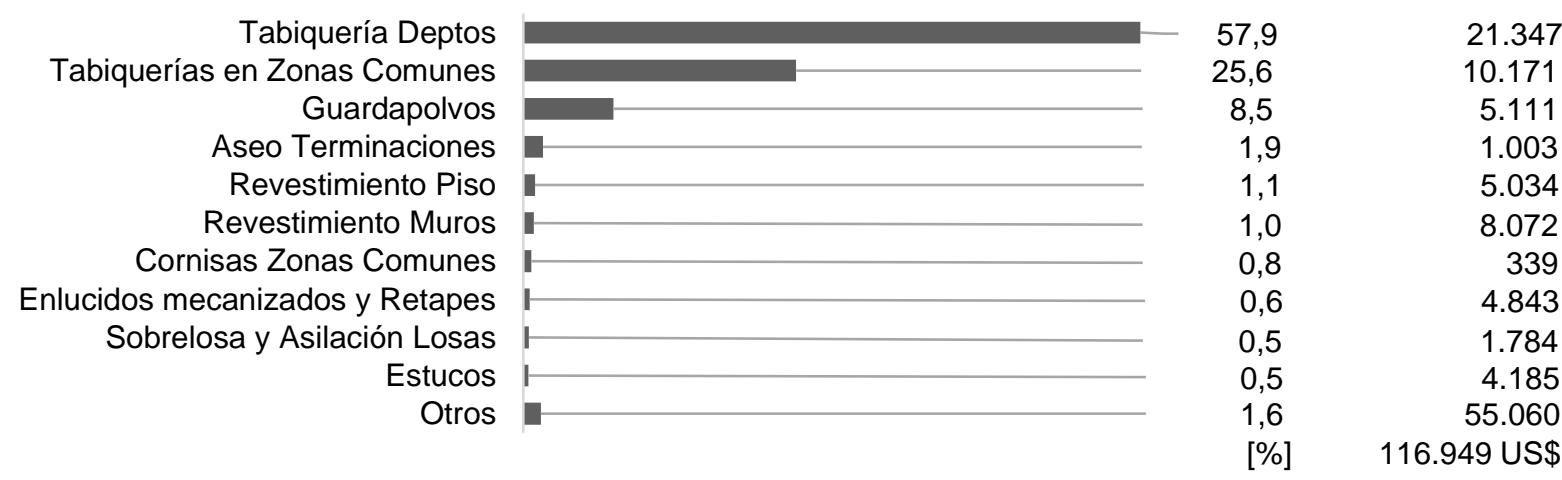

Fig. 4: Distribución residuos generados por partida en terminaciones.

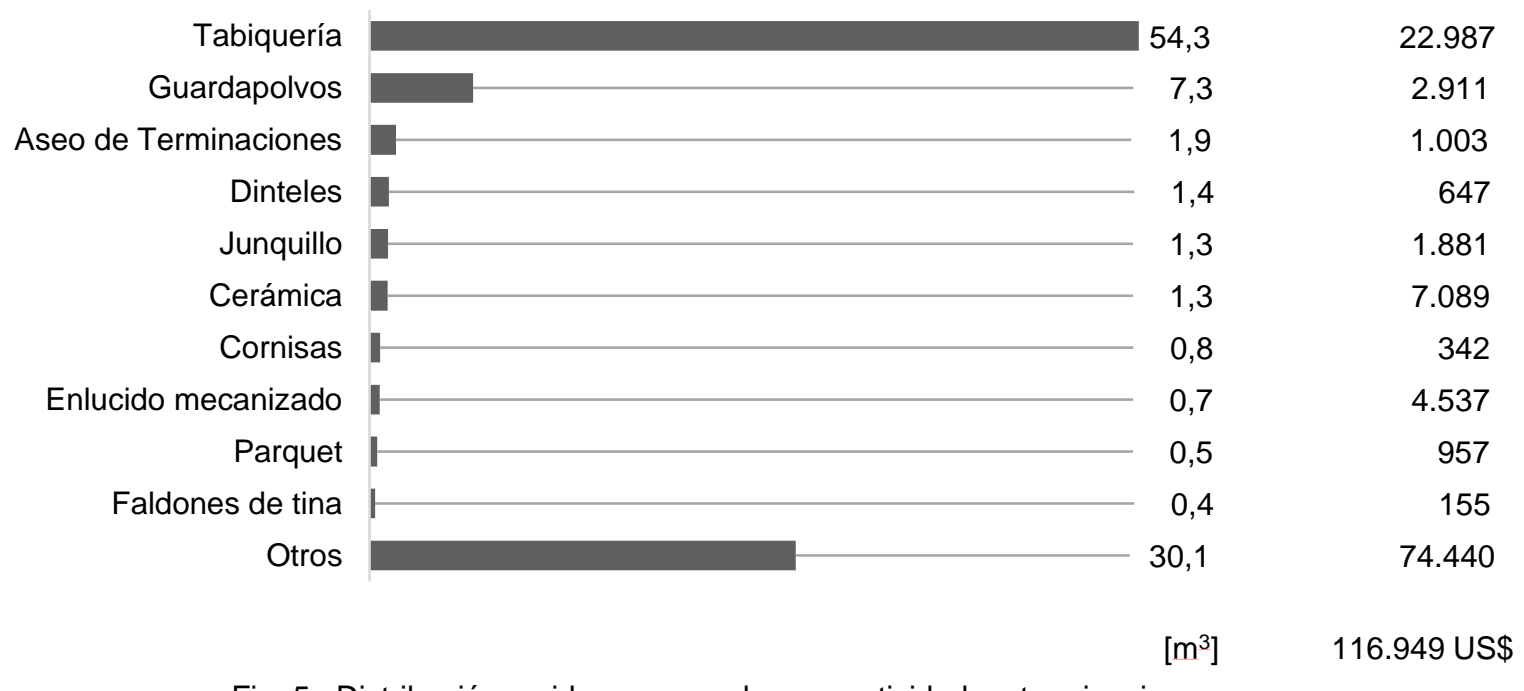

Fig. 5: Distribución residuos generados por actividad en terminaciones. 


\section{Visión General del Proyecto}

En lo que respecta a la visión general del proyecto, tal como se observa en la tabla 5 , los residuos esperados en la etapa de diseño alcanzan los $0,186 \mathrm{~m}^{3} / \mathrm{m}^{2}$. De este valor, $22 \%$ corresponden a residuos de Obra Gruesa y $78 \%$ a Terminaciones.

Tabla 5: Residuos generados totales e indicador de generación por m².

\begin{tabular}{|l|r|r|r|}
\hline \multicolumn{1}{|c|}{ Etapa } & Residuos generados $\left[\mathrm{m}^{3}\right]$ & Superficie útil $\left[\mathrm{m}^{2}\right]$ & Residuos $\left[\mathrm{m}^{3} / \mathrm{m}^{2}\right]$ \\
\hline Obra Gruesa & 544,79 & & 0,041 \\
\hline Terminaciones & $1.949,37$ & \multirow{2}{*}{$13.375,23$} & 0,146 \\
\cline { 1 - 2 } Total & $2.494,16$ & & 0,186 \\
\hline
\end{tabular}

La ponderación de las etapas Obra Gruesa y Terminaciones en los $\mathrm{m}^{3}$ de desechos difiere de la ponderación de las mismas en los costos de dichos desechos. En efecto, el costo del desecho considerando el precio de venta del elemento y el costo a botadero de los cubos totales (se considera el valor referencial de 9,46 US $\$ / \mathrm{m}^{3}$ como costo de transporte y disposición final) varía según tipo de desecho. Se estima que el costo de los residuos proyectados es US $\$ 188.234$ (ver tabla 6 ), de los cuales $38 \%$ corresponde a la etapa Obra Gruesa y $62 \%$ a Terminaciones.

Tabla 6: Costo de residuos generados diferenciados por etapa.

\begin{tabular}{|c|c|c|}
\hline Etapa & Residuos generados $\left[\mathrm{m}^{3}\right]$ & Costo [US\$] \\
\hline Obra Gruesa & 544,79 & $71.285,91$ \\
\hline Terminaciones & $1.949,37$ & $116.948,53$ \\
\hline Total & $2.494,16$ & $188.234,44$ \\
\hline
\end{tabular}

El costo proyectado de los residuos se divide en un $87 \%$ correspondiente al costo del material involucrado y un $13 \%$ asociado al costo de su disposición final (ver tabla 7). Asimismo, este costo representa el $1,19 \%$ del presupuesto del proyecto (ver tabla 8). Con lo cual, por cada $\mathrm{m}^{2}$ construido, se proyecta un costo de 14,1 US $\$ / \mathrm{m}^{2}$ por concepto de desechos, de este modo cada $\mathrm{m}^{3}$ de desecho tiene un valor promedio de 75,47 US $\$ / \mathrm{m}^{3}$ como se observa en la tabla 9.

Tabla 7: Costo total US\$ de residuos.

\begin{tabular}{|c|c|c|c|}
\hline $\begin{array}{c}\text { Volumen } \\
\text { Residuos }\left[\mathrm{m}^{3}\right]\end{array}$ & $\begin{array}{c}\text { Costo material según presupuesto } \\
\text { y pérdidas [US } \$]\end{array}$ & $\begin{array}{c}\text { Costo disposición final por } m^{3} \\
{[U S \$]}\end{array}$ & $\begin{array}{c}\text { Costo total } \\
{[U S \$]}\end{array}$ \\
\hline $2.494,16$ & $164.646,12$ & $23.588,32$ & $188.234,44$ \\
\hline
\end{tabular}

Tabla 8: Influencia de residuos en presupuesto.

\begin{tabular}{|c|c|c|}
\hline Costo Total [US\$] & Presupuesto proyecto [US\$] & Porcentaje del presupuesto \\
\hline $188.234,44$ & $15.774 .673,76$ & $1,19 \%$ \\
\hline
\end{tabular}

Tabla 9: Costos de residuos por unidad de superficie y volumen.

\begin{tabular}{|c|c|c|c|c|}
\hline Costo Total [US\$] & Superficie $\left[\mathrm{m}^{2}\right]$ & Costo $\left[\mathrm{US} \$ / \mathrm{m}^{2}\right]$ & Volumen Residuos $\left[\mathrm{m}^{3}\right]$ & Costo $\left[\mathrm{US} \$ / \mathrm{m}^{3}\right]$ \\
\hline $188.234,44$ & $13.375,23$ & 14,07 & $2.494,16$ & 75,47 \\
\hline
\end{tabular}

\section{DISCUSIÓN FINAL}

Las obras de construcción generan grandes cantidades de residuos urbanos en Chile con sus respectivos impactos económicos, sociales y ambientales. La reducción de los RCD son una prioridad si se desea satisfacer las actuales necesidades de la sociedad sin comprometer a las futuras generaciones la satisfacción de las suyas. La reducción de los RCD requiere comprender los procesos que los generan y los costos asociados a estos en los proyectos de construcción. En respuesta a la escasez de información al respecto, la presente investigación identifica la generación de residuos como un problema de productividad y entrega indicadores como el volumen de RCD generados por superficie construida y costo promedio de residuos. Lo anterior, proveen un insumo para inversionistas, evaluadores de proyectos $y$, además, provee una contribución a la literatura de RCD. 
En el presente caso de estudio, correspondiente a un proyecto de edificación en altura, de 21 pisos y uso residencial, se estima que se generarán $2.494,16 \mathrm{~m}^{3}$ de desechos. Asimismo, se estima que la etapa más influyente en la generación de residuos es Terminaciones, la que genera 3,56 veces los residuos que produce el de obra gruesa, su predecesora $\left(544,79 \mathrm{~m}^{3}\right.$ vs $1.949,37 \mathrm{~m}^{3}$ respectivamente). En la etapa de Terminaciones, las partidas que poseen mayor influencia en la generación de residuos son tabiques (o divisiones interiores) con $84 \%$ seguido por guardapolvos con un $8 \%$. Lo anterior obedece a que la partida tabiques engloba una gran cantidad de recursos que necesariamente deben ser reducidos en obra al momento de su colocación. Ejemplo de ello corresponden a: los montantes de acero galvanizado; la aislación con lana mineral y las planchas de yeso-cartón.

Al mismo tiempo, dentro de la etapa de Obra Gruesa, se evidencia que las partidas más influyentes en producción de residuos corresponden a hormigón con un 91\% aproximadamente y luego a partidas de techumbre y hojalatería, moldajes y enfierradura que sumadas alcanzan casi la totalidad del porcentaje restante (9\%). Esto se explica debido a que los volúmenes de hormigón son comparativamente superiores a los requeridos en otros materiales. De este modo, toda pérdida que sea dimensionada generará un gran volumen de residuos. En relación al volumen de residuos generados por superficie construida, se estimó para este caso de estudio $0,186 \mathrm{~m}^{3} / \mathrm{m}^{2}$ de desechos. Este resultado es coherente con el obtenido en el informe "Estudio preliminar residuos de la construcción en Antofagasta RESCON" de la Cámara Chilena de la Construcción Antofagasta (CCHC) donde se establecen $0,220 \mathrm{~m}^{3} / \mathrm{m}^{2}$ de desechos para las obras de edificación habitacional en altura analizadas (Cámara Chilena de la Construcción, 2013). Se justifica la comparación con dicho estudio, al considerar que se basó en 8 encuestas a empresas constructoras de Antofagasta (6) y Santiago (2), las cuales poseen la misma tipología del caso analizado e igual tecnología en procesos constructivos. Su diferencia se explica por la diferencia del alcance de los estudios, en donde el presente análisis corresponde a un estudio teórico.

Desde el punto de vista económico, se estimó un costo de $71.285,91$ US $\$(37,87 \%)$ para la etapa de Obra Gruesa y $116.948,53$ US\$ (62,13\%) para la etapa de Terminaciones. El costo total equivale a $188.234,44$ US\$ (100\%) y corresponde al 1,19\% del presupuesto del proyecto. El costo de disposición final de los recursos de materiales $(23.588,32$ US\$) representa el $12,53 \%$ del costo total. De igual forma, los costos por superficie construida y volumen de residuo son $14,07 \mathrm{US} \$ / \mathrm{m}^{2}$ y $75,47 \mathrm{US} \$ / \mathrm{m}^{3}$. Finalmente se destaca que la presente investigación proporciona un aporte a la temática al mostrar los volúmenes y costos de residuos por unidad de superficie construida y volumen de desecho generado. Sus limitaciones se basan exclusivamente en la información levantada desde el proyecto, ya que depende del orden, complejidad y pulcritud de la oficina de estudios quienes son los que entregan la información a clasificar.

\section{CONCLUSIONES}

De acuerdo al trabajo presentado y a los resultados obtenidos, se pueden plantear las siguientes conclusiones principales:

1.- La etapa más influyente en la generación de residuos es Terminaciones, la que genera 3,56 veces los residuos que produce el de obra gruesa, su predecesora (544,79 $\mathrm{m}^{3}$ vs $1.949,37 \mathrm{~m}^{3}$ respectivamente).

2.- En la etapa de Terminaciones, las partidas que poseen mayor influencia en la generación de residuos son tabiques (o divisiones interiores) con $84 \%$ seguido por guardapolvos con un $8 \%$.

3.- Dentro de la etapa de Obra Gruesa, se evidencia que las partidas más influyentes en producción de residuos corresponden a hormigón con un $91 \%$ aproximadamente y luego a partidas de techumbre y hojalatería, moldajes y enfierradura.

4.- El volumen de residuos generados por superficie construida, para este caso de estudio es de $0,186 \mathrm{~m}^{3} / \mathrm{m}^{2}$ de RC\&D con un costo total de $188.234,44$ US\$ correspondiente al $1,19 \%$ del presupuesto.

\section{5.- El costo ponderado de la gestión del RC\&D se estimó en 75,47 US $\$ / \mathrm{m}^{3}$.}

\section{REFERENCIAS}

Aldana, J., y A. Serpell, Temas y tendencias sobre residuos de construcción y demolición: Un metaanálisis, doi: 10.4067/S0718-915X2012000200002, Revista de la Construcción, 11(2), 4-16 (2012)

Cámara Chilena de la Construcción - Unidad de Estudios Antofagasta, Estudio preliminar residuos de la construcción en Antofagasta RESCON, 41p (2013)

Castaño, J., R. Misle-Rodriguez y otros tres autores, Gestión de residuos de construcción y demolición (RCD) en Bogotá: perspectivas y limitantes; doi: 10.14483/udistrital.jour.tecnura.2013.4.a09, Tecnura, 17(38), 121-129 (2013) 
Castillo, E. y F. Ossio, Waste Construction Management in Social Housing Projects, PLEA2012 - 28th Conference, Opportunities, Limits and Needs Towards an environmentally responsible architecture, Lima-Perú, 7-9 noviembre, (2012)

Gobierno de España, Conserjería de medio ambiente y ordenación del territorio. Secretaria Sectorial de Desarrollo sostenible y protección del medio ambiente. Dirección General de calidad ambiental. Servicio de Vigilancia de violencia e inspección ambiental. Lista europea de Residuos (LER). Orden MAM/304/2002, de 8 de febrero por la que se publican las operaciones de valorización y eliminación de residuos y la lista Europea de residuos. 40p (2002)

Gobierno de Chile, Ministerio del Medio Ambiente, Programación (2010-2014)

Jin, R., B. Li y otros tres autores, An empirical study of perceptions towards construction and demolition waste recycling and reuse in China, doi: 10.1016/j.resconrec.2017.07.034, Resources, Conservation and Recycling, 126, 86-98 (2017)

Romnée, A. y J. Vrijders, Innovative waste management practices on construction sites in Brussels: Lessons learned from pilots, HISER International Conference: Advances in recycling and management of construction and demolition waste, 340-344, Delft - Nueva Zelandia, 21-23 de Junio (2017)

Solís-Guzmán, J., M. Marrero, M.V. Montes-Delgado y A. Ramírez-de-Arellano, A spanish model for quantification and management of construction waste, doi:10.1016/j.wasman.2009.05.009, Waste Management, 29(9), 2542-2548 (2009)

Subramaniam, S., A. H. Abdullah, S. Nagapan, K. Kupusamy, H. Manian y Z. Daud, Investigate How Construction Waste Generation Rate is Different for Every Types of Project in Peninsular Malaysia using Site Visit Method, doi: 10.30880/ijie.2018.10.01.022, International Journal of Integrated Engineering, 10(1), 150-156 (2018)

Tam, V.W.Y. y C.M. Tam, Evaluations of existing waste recycling methods: A Hong Kong study, doi: 10.1016/j.buildenv.2005.06.017, Building and Environment, 41(12), 1649-1660 (2006)

Trachte, S., Gestion des déchets de chantier: Potentiel d'avenir pour le secteur de la construction en Région de bruxellesCapitale, Tesis de Doctorado, Université catholique de Louvain, Architecture et Climat (2003)

Umar, U., N. Shafiq y M. Hasnain-Isa, Investigation of construction wastes generated in the Malaysian residential sector, doi: 10.1177/0734242X18790359, Waste Management \& Research, 1-9 (2018)

Valdés, G. y J. Rapimán, Propiedades físicas y mecánicas de bloques de hormigón compuestos con áridos reciclados, Información Tecnológica, 18(3), 81-88 (2007)

Wu, Z., A.T.W. Yu, L. Shen y G. Liu, Quantifying construction and demolition waste: An analytical review, doi: 10.1016/j.wasman.2014.05.010, Waste Management, 34(9), 1683-1692 (2014) 
